\title{
INTERPOL ATING SEQUENCES ON CONVEX CURVES IN THE OPEN UNIT DISC
}

\author{
DENNIS H. WORTMAN 1
}

ABSTRACT. Let $D$ be the open unit disc in the complex plane, and let $C$ be the unit circle. Given a convex curve $\Gamma$ in $D \cup C$, internally tangent to $C$ at one point, then a sequence on $r$, successive points of which are equally spaced in the hyperbolic (Poincaré) metric, is shown to be interpolating. This result is then applied to the study of the Banach algebra $H^{\infty}$. The Gleason part of a point in the maximal ideal space of $H^{\infty}$ which lies in the closure of a convex curve in $D$ is proved to be nontrivial. In addition, for each point $m$ in the maximal ideal space of $H^{\infty}$ which lies in the closure of a compact subset of $D$ union a point of $C$, an interpolating $\mathrm{Bl}$ aschke product is constructed whose extension to the maximal ideal space has modulus less than 1 on $m$, and the relevance of this to the Shilov boundary of $H^{\infty}$ is discussed.

1. Introduction. A well-known result, arrived at independently by $\mathbb{W} . K$. Hayman and D. J. Newman, gives a relatively simple geometric condition under which a sequence in the open unit disc, lying on a ray from 0 to a point of the unit circle, is an interpolating sequence [2, p.203]. The purpose of this paper is to establish a similar condition for the case of a sequence, lying on a convex curve, internally tangent to the unit circle, and then to apply this result to the study of the Banach algebra, $H^{\infty}$, of all bounded analytic functions on the open unit disc.

Let $D$ be the open unit disc in the complex plane, and let $C$ be the unit circle. The geometry of $D$, as related to the study of many properties of $H^{\infty}$, is most advantageously expressed in terms of the pseudohyperbolic metric $\rho$, defined by

$$
\rho(\alpha, \beta)=\left|\frac{\alpha-\beta}{1-\bar{\alpha} \beta}\right|, \quad \alpha, \beta \in D .
$$

Presented to the Society, January 18, 1974; received by the editors March 28, 1973 and, in revised form, December 26, 1973.

AMS (MOS) subject classifications (1970). Primary 30A78, 46J15.

Key words and phrases. Interpolating sequence, convex curve, pseudohyperbolic metric, $H^{\infty}$, maximal ideal space, Gle ason part, Shilov boundary.

1 Portions of this paper constitute a part of the author's doctoral dissertation at the Massachusetts Institute of Technology. During his stay at M. I. T., the author was supported, in part, by a National Science Foundation Graduate Fellowship. 
In particular, the condition

$$
\inf _{n} \prod_{k \neq n} \rho\left(\alpha_{n}, \alpha_{k}\right)>0
$$

was shown by L. Carleson to characterize those sequences $\left\{a_{n}\right\}$ in $D$ on which interpolation of bounded sequences by functions in $H^{\infty}$ can always be obtained [2, p. 196]. As a consequence, a sequence $\left\{\alpha_{n}\right\}$ satisfying (C) is usually called an interpolating sequence, although, as in this paper and also in [3], the condition ( $C$ ), rather than the equivalent interpolative property, is the focus of interest. An interpolating sequence $\left\{\alpha_{n}\right\}$ is, of necessity, a Blaschke sequence, that is, the zero sequence of a function in $H^{\infty}$, and thus, corresponding to such a sequence is an (interpolating) Blaschke product $A(z)$, defined by

$$
A(z)=\prod_{n} \frac{\bar{\alpha}_{n}}{\left|\alpha_{n}\right| 1-\alpha_{n}-z} .
$$

$A(z)$ converges uniformly on compact subsets of $D$ and is bounded in modulus (by 1 ) and so is an element of $H^{\infty}$ (with sup norm 1 ).

We list, fri reference, the following easily verified properties of $\rho$ on $D$ :

(1) $\rho(\alpha, \beta) \geq \rho(|\alpha|,|\beta|)$.

(2) For a fixed $r, 0 \leq r<1, \rho\left(r, r e^{i \theta}\right)$ is a monotone increasing function of $\theta, 0 \leq \theta \leq \pi$.

(3) If $\Gamma$ is a convex, decreasing curve in $D \cup C$ (as a function $y=\Gamma(x)$ ), intersecting $C$ at 1 , then for $\alpha, \beta, \gamma \in \Gamma$, with $|\alpha|<|\beta|<|\gamma|$, $\rho(\alpha, \beta)<\rho(\alpha, \gamma)$.

Under certain circumstances, condition (C ) may be replaced by somewhat simpler conditions. Thus the result of Hayman and Newman, referred to previously, implies that a sequence $\left\{a_{n}\right\}$ of points, of increasing modulus, on a ray from 0 to a point of $C$, is interpolating if and only if

$$
\inf _{n} \rho\left(\alpha_{n}, \alpha_{n+1}\right)>0
$$

that is, there is a minimal (positive) distance, in the pseudohyperbolic metric, between successive points. An extension of their arguments yields the same result for a sequence, of increasing modulus, in a Stolz angle, formed by two rays from a point on $C$ (nontangential approach to $C$ ). In $\S 2$, it is shown that (HN) is also a necessary and sufficient condition for a sequence $\left\{a_{n}\right\}$, approaching $C$ tangentially on a convex curve in $D$, to be interpolating. This follows as a corollary of the main result of that section which shows that sequences on convex curves tangent to $C$, successive 
terms of which are equally spaced in the pseudohyperbolic metric, are interpolating. Then in $\$ 3$, this result is applied to the study of the structure of the maximal ideal space $\mathbb{M}$, of $H^{\infty}$. The Gleason part of a point in $\mathbb{M}$, lying in the closure of a convex curve tangent to $C$, is shown to be nontrivial; and a generalization of a theorem of Beurling, proved by M. Weiss in [4], is obtained, and its relation to the Shilov boundary of $H^{\infty}$ is discussed.

I wish to thank Professor K. Hoffman for many useful discussions and helpful suggestions concerning the content and format of this paper. His book [2] provides an excellent introduction to and reference for the study of the Banach algebra $H^{\infty}$.

2. Interpolating sequences on convex curves. The fundamental result in this paper is the following theorem.

Theorem 1. Let $\Gamma$ be a convex curve in $D \cup C$, with $\Gamma \cap C=\{1\}$ and $\Gamma$ tangent to $C$ at 1. Let $\left\{\alpha_{n}\right\}$ be a sequence of points on $\Gamma \backslash\{1\}$, with $\left|\alpha_{n}\right| \uparrow 1$ and $\rho\left(\alpha_{n}, a_{n+1}\right)=\delta, n=1,2,3, \cdots$, for some number $\delta, 0<\delta<1$. Then $\left\{\alpha_{n}\right\}$ is an interpolating sequence.

Before proving Theorem 1, we state and prove the following geometric lemma which will be used in that proof.

Lemma. Let $\Gamma$ be as in Theorem 1. Let $\delta$ be a number, $0<\delta<1$. Let $z$ be an arbitrary point on $\Gamma$, and let $w$ be the point on $\Gamma$ satisfying: arg $w>$ $\arg z$ and $\rho(u, z)=\delta$. Let $v$ be the point in $D$ satisfying: $\arg v=\arg w$ and $|v|=|z|$. Then $\lim \inf _{|z| \rightarrow 1} \rho(z, v) \geq \delta$.

Proof. Geometrically the picture is as follows. $z$ is a (variable) point on $\Gamma$ and $u$ is the point on $\Gamma$ which is $\delta \rho$-units "behind" $z$. The Lemma states that the point $\nu$, determined by the argument of $w$ and the modulus of $z$, lies outside the $\rho$-disc of radius $\delta$, centered at $z$, if $z$ is "near" 1 , in the usual metric on $D$.

Thus, let $z, w$, and $v$ be as described in the Lemma, with (polar) notations $z=r e^{i \theta}, w=s e^{i \phi}, v=r e^{i \phi} . w$ and $v$, of course, depend on $z$ (and on $\Gamma$ ), as will the other points and quantities introduced below.

Let $\zeta=r e^{i \psi}$ be the point in $D$ satisfying: $\arg \zeta>\arg z,|\zeta|=|z|$, and $\rho(z, \zeta)=\delta$. The proof will be complete if we can show that (eventually)

$$
\phi=\arg v=\arg w \geq \arg \zeta=\psi,
$$

for then $\rho(z, v) \geq \rho(z, \zeta)=\delta$ by (Rं2).

Let $\xi=t e^{i \psi}$ be the point in $D$ satisfying: $\arg \xi=\arg \zeta,|\xi|<|z|$, and $\rho(z, \xi)=\delta$. 
Let $m$ be the slope of the line joining $u$ and $z$, and let $m^{\prime}$ be the slope of the line joining $\xi$ and $z$. Then (1) is equivalent to saying that $w$ lies between $\xi$ and $\zeta$ on the $\rho$-circle of radius $\delta$, centered at $z$, or

$$
m<m^{\prime} \text {. }
$$

Note that, by the convexity of $\Gamma$ and its tangency to $C$ at $1, m \rightarrow-\infty$ as $z \rightarrow 1$. We will conclude the proof by showing that $m^{\prime}$ is bounded from below as $z \rightarrow 1$.

An examination (by tedious computation) of the pseudohyperbolic distances between the points $w, \zeta, \xi$ and $z$ yields that

$$
t=\left(r^{2}-\delta^{2}\right) / r\left(1-r^{2} \delta^{2}\right), \quad t-r=-\delta^{2}\left(1-r^{2}\right) / r\left(1-r^{2} \delta^{2}\right),
$$

$$
1-\cos (\psi-\theta)=\delta^{2}\left(1-r^{2}\right)^{2} /\left(1-\delta^{2}\right) 2 r^{2} .
$$

Since

$$
m^{\prime}=(t \sin \psi-r \sin \theta) /(t \cos \psi-r \cos \theta)
$$

using (3), and noting that $1-\cos (\psi-\theta)$ is asymptotic to $1 / 2(\psi-\theta)^{2}$ as $r=|z| \rightarrow 1$, we find that

$$
\begin{aligned}
\liminf _{r \rightarrow 1} m^{\prime} & =\lim _{r \rightarrow 1} \inf \frac{t \psi-r \theta}{(t-r)+1 / 2\left(r \theta^{2}-t \psi^{2}\right)} \\
& =\liminf _{r \rightarrow 1} \frac{(t-r) \theta+t(\psi-\theta)}{(t-r)\left(1-1 / 2 \theta^{2}\right)+\theta(\psi-\theta)+1 / 2(\psi-\theta)^{2}} \\
& =-\left(1-\delta^{2}\right)^{1 / 2} / 2 \delta .
\end{aligned}
$$

Proof of Theorem 1 . We may assume that $\Gamma$ is actually a monotone decreasing curve (as a function $y=\Gamma(x)$ of real variables) lying in the first quadrant of the $x-y$ plane. We may then write $\alpha_{n}=r_{n} \exp \left(i \theta_{n}\right), n=1,2$, $\cdots$, where $r_{n}>0$ and $0<\theta_{n}<\pi / 2$. Thus $r_{n} \nearrow 1$ and $\theta_{n} \searrow 0$ as $n \rightarrow \infty$.

Property (R3) implies that $\rho\left(\alpha_{n}, \alpha_{k}\right) \geq \delta$ if $k \neq n$. Consequently, to prove that $\left\{a_{n}\right\}$ satisfies condition (C), it is equivalent to show that $\left\{a_{n}\right\}$ satisfies

$$
\sup _{n} \sum_{k \neq n}\left[1-\rho^{2}\left(\alpha_{n}, \alpha_{k}\right)\right]<\infty \quad[3, \text { p. } 9]
$$

The key step in verifying $\left(C^{\prime}\right)$ will be to relate the pseudohyperbolic distance between successive points in the sequence $\left\{a_{n}\right\}$ to the angular separation between the points. It is here that the preceding Lemma will 
be applied to replace $\rho\left(\alpha_{k}, \alpha_{k+1}\right)$ by $\rho\left(\beta_{k}, \alpha_{k+1}\right)$, where $\beta_{k}$ is the point obtained by translating $\alpha_{k}$ along the ray $\theta=\arg \alpha_{k}$ to the point whose modulus is $\left|a_{k+1}\right|$.

Thus, consider a (fixed) $n$. Let $\phi_{k}=\theta_{n}-\theta_{k}, k=1,2,3, \cdots$, be the angle between $\alpha_{n}$ and $\alpha_{k}$. We note that

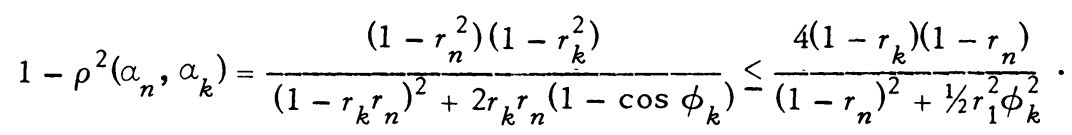

Combining this with $\left(C^{\prime}\right)$, we need now show only that

$$
\sup _{n} \sum_{k \neq n} a_{k}(n)<\infty,
$$

where

$$
a_{k}(n)=\left(1-r_{k}\right)\left(1-r_{n}\right) /\left(\left(1-r_{n}\right)^{2}+1 / 2 r_{1}^{2} \phi_{k}^{2}\right), \quad k=1,2, \cdots .
$$

We now relate $\left(1-r_{k}\right)$ to the angular separation between $\alpha_{k-1}$ and $\alpha_{k}$. Thus let $\Delta \phi_{k}=\phi_{k}-\phi_{k-1}, k=2,3, \cdots$. Applying the Lemma to the sequence $\left\{\alpha_{n}\right\}$, we find that for large $k$, say $k \geq N$,

$$
\delta=\rho\left(\alpha_{k-1}, \alpha_{k}\right) \leq \rho\left(\beta_{k}, \alpha_{k}\right), \quad \beta_{k}=r_{k} \exp \left(i \theta_{k-1}\right), k=2,3, \cdots .
$$

In addition, since

$$
\rho^{2}\left(\beta_{k}, \alpha_{k}\right)=\frac{2 r_{k}^{2}\left(1-\cos \Delta \phi_{k}\right)}{\left(1-r_{k}^{2}\right)^{2}+2 r_{k}^{2}\left(1-\cos \Delta \phi_{k}\right)}, \quad k=2,3, \cdots,
$$

we have

$$
\rho\left(\beta_{k}, \alpha_{k}\right) \leq \Delta \phi_{k} /\left(1-r_{k}^{2}\right), \quad k=2,3, \cdots .
$$

Therefore, combining (1) and (2), we conclude that there exists a positive number $c$, such that

$$
\delta \leq c \Delta \phi_{k} /\left(1-r_{k}^{2}\right), \quad k=2,3, \cdots .
$$

To verify (C"), we now consider two cases: (i) $\Sigma_{k<n} a_{k}(n)$ and (ii) $\Sigma_{k>n} a_{k}(n)$.

Case (i). (Points to the "left" of $\alpha_{n}$.)

We will estimate (i) by a definite integral, but it will first be necessary to translate angles. Let $\left\{R_{m}\right\}$ be a sequence of positive numbers monotonely increasing to 1 , for which $\rho\left(R_{m-1}, R_{m}\right) \geq \delta$ and

$$
0<c_{1}<\left(1-R_{m}\right) /\left(1-R_{m-1}\right)<c_{2}<1, \quad m=2,3, \cdots,
$$


for some numbers $c_{1}$ and $c_{2}$. [If $\delta<(j-1) /(j+1)$, then one choice for $R_{m}$ is $\left.R_{m}=1-j^{-m}, m=1,2, \cdots.\right]$ Since, by (R 1$)$,

$$
\delta=\rho\left(\alpha_{k}, \alpha_{k+1}\right) \geq \rho\left(\left|\alpha_{k}\right|,\left|\alpha_{k+1}\right|\right), \quad k=1,2, \cdots,
$$

$\left|\alpha_{k}\right|$ and $\left|\alpha_{k+1}\right|$ either lie between two successive terms of $\left\{R_{m}\right\}$ or are separated by exactly one such term. In either case, it follows from (3) and (4) that

$$
1-r_{k} \leq\left(1-r_{k+1}\right) / c_{1}^{2}<c \Delta \phi_{k+1} / c_{1}^{2} \delta, \quad k=1,2, \cdots .
$$

Hence, if we set $p=2^{1 / 2} c / c_{1}^{2} \delta r_{1}$, and use (5), we find that

$$
\begin{aligned}
\sum_{k<n} a_{k}(n) & \leq p \sum_{k<n} \frac{1-r_{n}}{\left(1-r_{n}\right)^{2}+1 / 2 r_{1}^{2} \phi_{k}^{2}}\left(r_{1} \Delta \phi_{k+1} / 2^{1 / 2}\right) \\
& \leq p \int_{0}^{\infty} \frac{1-r_{n}}{\left(1-r_{n}\right)^{2}+x^{2}} d x=\pi p / 2 .
\end{aligned}
$$

Case (ii). (Points to the "right" of $a_{n}$.)

By an argument similar to that in Case (i), but without the need to translate angles in order to obtain the integral estimate, we find by setting $q=2^{1 / 2} c / \delta r_{1}$ that $\Sigma_{k>n} a_{k}(n) \leq 1 / 2 \pi q$.

Thus, by combining Cases (i) and (ii), we see that $\left\{a_{n}\right\}$ satisfies ( $C^{\prime \prime}$ ) since $p$ and $q$ are independent of $n$, and so $\left\{a_{n}\right\}$ is an interpolating sequence.

The following corollary extends the Hayman-Newman theorem to the case of sequences approaching $C$ tangentially on a convex curve.

Corollary. Let $\Gamma$ be a convex curve in $D \cup C$, with $\Gamma \cap C=\{1\}$ and $\Gamma$ tangent to $C$ at 1. Let $\left\{a_{n}\right\}$ be a sequence of points on $\Gamma \backslash\{1\}$, with $\left|a_{n}\right| \nearrow 1$. Then $\left\{\alpha_{n}\right\}$ is an interpolating sequence if and only if $\left\{\alpha_{n}\right\}$ satisfies (HN); i.e., $\inf _{n} \rho\left(\alpha_{n}, \alpha_{n+1}\right)>0$.

Proof. The necessity of (HN) follows immediately from (C).

The proof of Theorem 1 also proves the sufficiency of (HN). In fact, only in obtaining ( 5 ) in the proof of Theorem 1 was it necessary to have $\delta=\rho\left(a_{k}, a_{k+1}\right)$ rather than $\delta \leq \rho\left(a_{k}, a_{k+1}\right)$. However, (5) for the latter condition above follows directly from (5) for the former condition. For, if $\delta<\rho\left(\alpha_{k}, a_{k+1}\right)$, let $\alpha_{k}^{i}$ be the point on $\Gamma$ between $\alpha_{k}$ and $\alpha_{k+1}$ for which $\rho\left(\alpha_{k}, \alpha_{k}^{\prime}\right)=\delta$. Then, by the convexity of $\Gamma$, the angle between $\alpha_{k}$ and $\alpha_{k}^{\prime}$ is less than the angle between $\alpha_{k}$ and $\alpha_{k+1},\left(\Delta \phi_{k+1}\right)$, and thus (5) is valid for the condition $\delta<\rho\left(\alpha_{k}, \alpha_{k+1}\right)$ also. 
3. Applications to $H^{\infty}$. Let $\mathbb{R}$ denote the maximal ideal space of $H^{\infty}$, and $M_{1}$ the fiber of $M$ over 1 ; i.e., the subset of $M$ consisting of all homomorphisms $m$ whose value on the identity function $z$ is 1 . In this section we will examine properties of the structure of $\mathbb{M}_{1}$ related to convex subsets of $D$.

Theorem 2. Let $\Gamma$ be a convex curve in $D \cup C$, with $\Gamma \cap C=\{1\}$ and

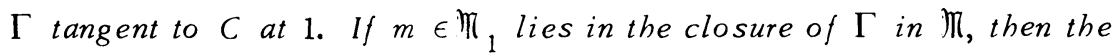
Gleason part of $m$ is nontrivial.

Proof. Let $\left\{\beta_{i}\right\}$ be a net, $\beta_{i} \in \Gamma$, such that $\lim _{i} \beta_{i}=m$. Fix a number $\delta, 0<\delta<1$, and form a sequence $\left\{a_{n}\right\}$ satisfying the hypotheses of Theorem $1,\left|a_{n}\right| / 1$ and $\rho\left(\alpha_{n}, \alpha_{n+1}\right)=\delta$. Let $\alpha_{n(i)}$ be a member of $\left\{\alpha_{n}\right\}$ which is nearest to $\beta_{i}$ in the metric $\rho$. Thus, eventually, $\rho\left(\alpha_{n(i)}, \beta_{i}\right)<\delta$. Passing to subnets, we may assume that $\alpha_{n(i)} \rightarrow m^{\prime} \in \mathbb{M}_{1}$. Hence, by the lower semicontinuity of $\rho$ on $M \times M\left[3\right.$, p. 103], $\rho\left(m^{\prime}, m\right)<\delta<1$, so the Gleason part of $m$ is nontrivial, since it is thus the same as that of $m^{\prime}$ which is nontrivial by $[3, \mathrm{p} .88]$.

As a second application of Theorem 1, we prove

Theorem 3. Let $K$ be a compact subset of $D \cup C$, with $K \cap C=\{1\}$. Then there is an interpolating sequence $\left\{\alpha_{n}\right\}$ in $D$, with $\alpha_{n} \rightarrow 1$, and for which the associated (interpolating) Blaschke product $A(z)$ is of sup norm less than 1 on $K \backslash\{1\}$ : $\sup \{|A(z)|: z \in K \backslash\{1\}\}<1$.

Proof. Since we may replace $K$ by the closed convex hull of $K \cup \bar{K}$, where $\bar{K}=\{\bar{z}: z \in K\}$, we may assume that $K$ is a compact, convex subset of $D \cup C$, symmetric about the real axis, and having as a boundary a curve $\gamma$ which is tangent to $C$ at 1 . (If $K \cup \bar{K}$ is contained in a Stolz angle, it may be replaced by a disc tangent to $C$ at 1.) Let $\Gamma$ denote the portion of $\gamma$ lying above the real axis, and form a sequence $\left\{\alpha_{n}\right\}$ on $\Gamma$, satisfying the conditions of Theorem 1 , using any number $\delta, 0<\delta<1$. Then the sequence $\left\{a_{1}, \bar{\alpha}_{1}, \cdots, a_{n}, \bar{\alpha}_{n}, \cdots\right\}$ is an interpolating sequence, as the union of two such sequences, and the corresponding Blaschke product $A(z)$ has the property that $|A(z)|<\delta$ for $z$ on that portion of $\gamma$ "between" $\alpha_{1}$ and 1 or "betwe en" $\bar{\alpha}_{1}$ and 1 , since such $z$ are within $\delta \rho$-units of some $a_{n}$ or $\bar{a}_{n}$. And so, because the remaining portion of $\gamma$ is a positive distance from $C$, there is a number $d, 0<d<1$, such that $|A(z)|<d$ for all $z \in \gamma \backslash\{1\}$. The Phragmén-Lindelöf theorem then implies that 


$$
\sup \{|A(z)|: z \in K \backslash\{1\}\}=\sup \{|A(z)|: z \in \gamma \backslash\{1\}\},
$$

and the result follows.

Corollary. Let $K$ be a compact subset of $D \cup C$, with $K \cap C=\{1\}$. If $m \in \mathbb{M}_{1}$ lies in the closure of $K \backslash\{1\}$ in $\mathbb{M}$, then there is an interpolating Blaschke product $A(z)$ whose extension $\hat{A}$ to $\mathbb{M}$ is of modulus less than 1 on $m:|\hat{A}(m)|<1$.

Theorem 3 contains a theorem of Beurling [4], which arose from a query of $\mathrm{M}$. Weiss about the relation of $\mathrm{Blaschke}$ sequences to the interior of $\mathrm{M}_{1}$ in $M \backslash D$. The corollary to it provides a partial solution to a question posed by $\mathrm{K}$. Hoffman concerning the relation of interpolating Blaschke products to the Shilov boundary $\partial$ of $H^{\infty}$. By a well-known theorem of D. J. Ne wman [2, p. 179], $\partial$ consists of those homomorphisms of $H^{\infty}$ on which (the extensions of ) all Blaschke products have modulus 1 . If we denote by 9 the set of all homomorphisms of $H^{\infty}$ on which (the extensions of). all interpolating Blaschke products have modulus 1 , then by Newman's theorem, $\partial \subseteq 9$. Is $\partial=9$ ? The corollary to Theorem 3 shows that a point in $\mathbb{R} \backslash D$ which lies in the closure of a compact subset of $D$ union a point of $C$ is not in 9 . Of course, this is only a partial answer to the question. But, if $\partial \neq 9$, then the sup norm algebra $\hat{H}^{\infty} \mathrm{lg}_{\mathrm{g}}$ is an example of a proper sup norm algebra which has only trivial Gleason parts. The first example of such a phenomenon was given by $B$. Cole [1].

\section{REFERENCES}

1. B. Cole, One point parts and the peak point conjecture, Ph.D. Dissertation, Yale University, New Haven, Conn., 1968.

2. K. Hoffman, Banach spaces of analytic functions, Prentice-Hall Ser. in Modern Analysis, Prentice-Hall, Englewood Cliffs, N.J., 1962. MR 24 \#A2844.

3. - Bounded analytic functions and Gleason parts, Ann. of Math. (2) 86 (1967), 74-111. MR 35 \#5945.

4. M. L. Weiss, Note on a theorem of Beurling, Proc. Amer . Math. Soc. 19 (1968), 793-795. MR 37 \#3006.

DEPARTMENT OF MATHEMATICS, UNIVERSITY OF MASSACHUSETTS, BOSTON, MASSACHUSETTS 02125 